\title{
Design Considerations for Pipelined Continuous-Time Incremental Sigma-Delta ADCs
}

\author{
Sha Tao, Jiazuo Chi, and Ana Rusu \\ School of ICT, KTH Royal Institute of Technology \\ SE-164 40 Kista, Stockholm, Sweden \\ Email: stao@kth.se
}

\begin{abstract}
This paper addresses design considerations for power-efficient pipelined continuous-time (CT) incremental Sigma-Delta $(I \Sigma \Delta)$ ADC architectures. By pipelining identical CT I $\Sigma \Delta$ ADC stages, the proposed architecture provides the design freedom coming from both the pipeline ADC and the I $\Sigma \Delta$ ADC. In searching for a low-power solution given a target resolution, different configurations are examined analytically and simulated using behavioral models. For further power reduction, power-efficient circuits are proposed to implement the active blocks in each configuration. Based on the architecture-level analysis, a configuration that leads to minimum power-area consumption is chosen and implemented as a test-case using the proposed circuit blocks. Post-layout simulations show that the test-case ADC, with 3.2-kHz bandwidth, achieves a peak SNDR of 82.5-dB while dissipating a total power of 18.27- $\mu \mathrm{W}$.
\end{abstract}

\section{INTRODUCTION}

Incremental Sigma-Delta (I $\Sigma \Delta$ ) ADCs [1] have been recently investigated for ultra-low-power biosensor applications, which feature signal bandwidth from DC to $10-\mathrm{kHz}$ and resolution ranging from 9-bit to 14-bit. The restricted batterylife and chip area in biosensors impose challenges in designing power-efficient I $\Sigma \Delta$ ADCs that meet both the bandwidth and resolution specifications. High-order [2] and extended-range (ER) [3] I $\Sigma \Delta$ ADCs using discrete-time (DT) implementation have been proposed to reduce the number of cycles per conversion, leading to better power-efficiency than the firstorder I $\Sigma \Delta$ ADC. A high-order continuous-time (CT) I $\Sigma \Delta$ ADC [4] has been developed lately, aiming to take advantage of the relaxed settling and bandwidth requirements of active blocks in CT loop filters. This work takes a step further by proposing and implementing a pipelined CT I $\Sigma \Delta$ ADC while applying power-conscious, area-efficient design strategy at both architecture and circuit levels.

\section{Proposed Architecture}

In the proposed ADC architecture, shown in Fig. 1, $n$ stages of $\mathrm{CT} I \Sigma \Delta$ ADCs are cascaded and process each sample in a pipelining fashion. Each stage performs a sample-and-hold (SH) operation at the rate of $f_{s} / M$. The number of clock cycles per conversion, $M$, is equivalent to the oversampling ratio (OSR) in conventional $\Sigma \Delta$ ADCs. The CT I $\Sigma \Delta$ modulator in each stage processes each sample at an oversampling rate of $f_{s}$. After $M$ cycles, the residue (i.e., the quantization error) of each stage, $V_{\text {res }, i=\{1,2, \ldots n-1\}}$, is sampled and fed into the subsequent stage. At the same time, the digital filter produces

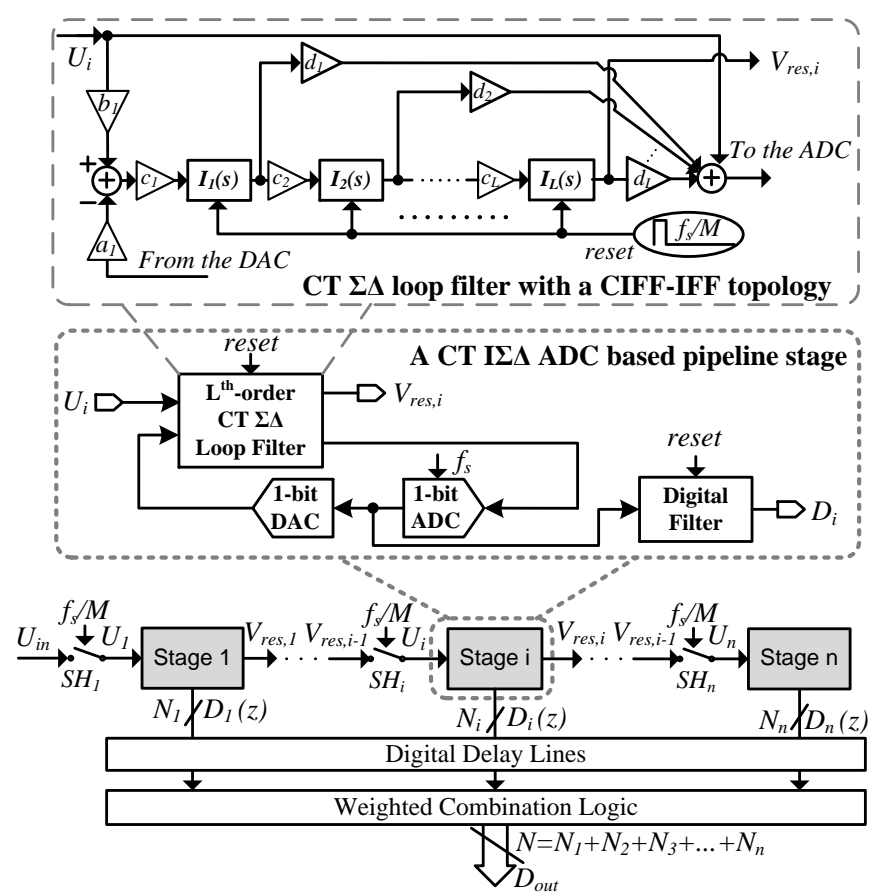

Fig. 1: Pipelined CT I $\Sigma \Delta$ ADC.

a valid result, $D_{\text {out }, i=\{1,2, \ldots n\}}$. The residue of each stage can be readily obtained at the $L^{\text {th }}$ integrator's output, when the CT $\Sigma \Delta$ loop filter is implemented with a CIFF-IFF topology. After the sample has propagated through all the stages, the valid digital outputs, $D_{1}(z) \ldots D_{n}(z)$, are combined to yield a final digital word, $D_{\text {out }}$. In the end, the loop filters as well as the digital filters, which have the transfer function as shown in [5], are reset and ready to accept the next sample.

Another feature that favors the proposed architecture is the inherent flexibility in achieving different conversion speed and resolution. It enables this architecture to accommodate various biopotential signals with different specifications of the ADC's bandwidth and dynamic range. The conversion rate can be tuned by altering the number of $M$ while maintaining the internal clock frequency $f_{s}$. Different conversion resolutions can be achieved by taking the digitized outputs from various numbers of stages in the combination logic. Compared to a conventional pipeline ADC stage, a CT I $\Sigma \Delta$ ADC stage has several advantages. By employing noise shaping and 
TABLE I: Different configurations of the proposed ADC architecture. [ $n$ : number of stages; $L$ : modulator order]

\begin{tabular}{lllllll}
\hline \hline Configuration & \multicolumn{2}{c}{$n=1$} & \multicolumn{2}{c}{$n=2$} & \multicolumn{2}{c}{$n=3$} \\
\hline$(n, L)$ & $(1,2)$ & $(1,3)$ & $(2,2)$ & $(2,3)$ & $(3,1)$ & $(3,2)$ \\
\hline \hline
\end{tabular}

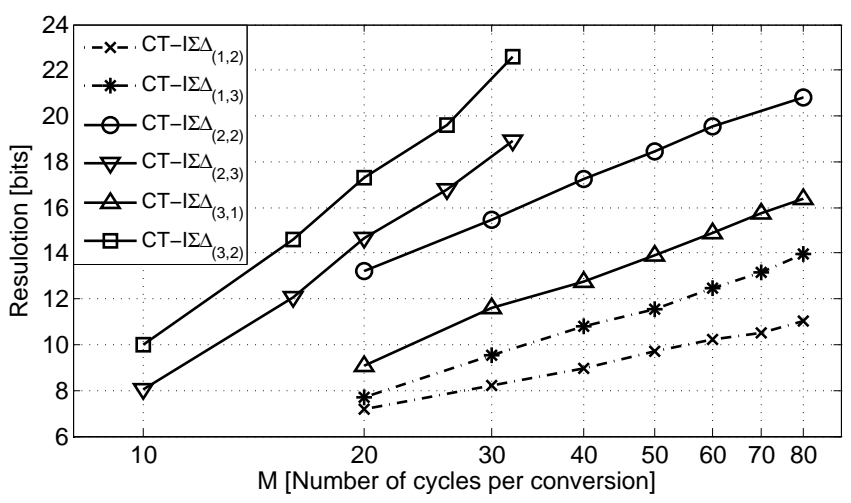

Fig. 2: Simulated resolution of different configurations of the proposed pipelined CT I $\Sigma \Delta$ ADC architecture.

oversampling, it achieves higher resolution with a 1-bit ADC, and hence avoids the need to implement a flash ADC and a multi-level DAC. Also, it does not require a subtractor for residue generation and a gain block for matching the input range of the subsequent stage. Therefore, it avoids a significant amount of efforts in counteracting the errors induced by the circuit non-idealities of these blocks.

Due to the pipelining operation, the ADC can achieve a resolution of $N=N_{1}+\cdots+N_{n}$ and its conversion rate is set by the time of a single stage, $M / f_{s}$. The theoretical resolution, $N$, can be estimated as a sum of the resolution achieved by each $L^{t h}$-order CT I $\Sigma \Delta$ ADC stage:

$N=\sum_{i=1}^{n} \log _{2}\left\{\left(\frac{U_{i, \max }}{V_{F S}} \frac{(M+L-1) !}{L !(M-1)}+1\right)\left(a_{1} \prod_{j=1}^{L} c_{j}\right)\right\}$,

where $U_{i, \max }$ is the peak amplitude of the input signal; $V_{F S}$ is the full-scale amplitude; and $b_{1}, c_{1 \ldots L}$ are the loop filter coefficients as shown in Fig. 1. The resolution derivation of a high-order CT I $\Sigma \Delta$ ADC can be found in [4]. Although each $D_{\text {out }}$ comes with a fixed conversion latency of $n \times M / f_{s}$, it is tolerable in most of the biosensor applications.

The presented pipelined CT I $\Sigma \Delta$ ADC architecture has three design parameters. i) the number of pipeline stages, $n$, ii) the number of cycles per conversion, $M$, and iii) the modulator order in each stage, $L$. Different combinations of $n$ and $L$ are configured for the proposed pipelined architecture with identical CT I $\Sigma \Delta$ ADC stages, as shown in Table I. Fig. 2 shows the simulated resolution of each configuration when the number of cycles $M$ is swept. It can be seen that a much higher theoretical resolution can be achieved without sacrificing the conversion rate, when $n \geq 2$ and $L \geq 2$. Larger $n$ and $L$, on the other hand, increases the circuit complexity which leads to
TABLE II: Specifications for the active blocks in each configuration.

\begin{tabular}{|c|c|c|c|c|c|c|}
\hline \multirow[t]{2}{*}{$n=2, L=2$} & \multicolumn{6}{|c|}{ 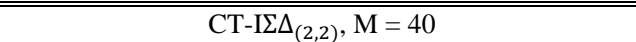 } \\
\hline & \multicolumn{2}{|c|}{$\mathrm{INT}_{11}$} & $\mathrm{INT}_{12}$ & \multicolumn{2}{|c|}{$\mathrm{INT}_{21}$} & $\mathrm{INT}_{22}$ \\
\hline GBW (k Hz) & \multicolumn{2}{|c|}{204.8} & 128 & 102.4 & \multicolumn{2}{|r|}{51.2} \\
\hline DC Gain [dB] & \multicolumn{2}{|l|}{40} & 30 & \multicolumn{2}{|c|}{30} & 20 \\
\hline SR $\left[10^{5} \mathrm{~V} / \mathrm{s}\right]$ & \multicolumn{2}{|c|}{2.05} & 1.28 & \multicolumn{2}{|c|}{1.02} & 0.51 \\
\hline \multirow{2}{*}{$\mathrm{n}=2, \mathrm{~L}=3$} & \multicolumn{6}{|c|}{$\mathrm{CT}-\mathrm{I} \Sigma \Delta_{(2,3)}, \mathrm{M}=26$} \\
\hline & $\mathrm{INT}_{11}$ & $\mathrm{INT}_{12}$ & $\mathrm{INT}_{13}$ & $\mathrm{INT}_{21}$ & $\mathrm{INT}_{22}$ & $\mathrm{INT}_{23}$ \\
\hline GBW (k Hz) & 133.12 & 66.56 & 33.28 & 83.2 & 33.28 & 16.64 \\
\hline DC Gain $[\mathrm{dB}]$ & 40 & 30 & 20 & 30 & 20 & 10 \\
\hline SR $\left[10^{5} \mathrm{~V} / \mathrm{s}\right]$ & 1.33 & 0.67 & 0.33 & 0.83 & 0.33 & 0.17 \\
\hline \multirow[t]{2}{*}{$n=3, L=1$} & \multicolumn{6}{|c|}{$\mathrm{CT}-\mathrm{I} \Sigma \Delta_{(3,1)}, \mathrm{M}=80$} \\
\hline & \multicolumn{2}{|c|}{$\mathrm{INT}_{11}$} & \multicolumn{2}{|c|}{$\mathrm{INT}_{21}$} & \multicolumn{2}{|c|}{$\mathrm{INT}_{31}$} \\
\hline GBW (k Hz) & \multicolumn{2}{|c|}{1024} & \multicolumn{2}{|c|}{512} & \multicolumn{2}{|c|}{128} \\
\hline DC Gain [dB] & \multicolumn{2}{|c|}{55} & \multicolumn{2}{|c|}{50} & \multicolumn{2}{|c|}{20} \\
\hline $\mathrm{SR}\left[10^{5} \mathrm{~V} / \mathrm{s}\right]$ & \multicolumn{2}{|c|}{10.24} & \multicolumn{2}{|c|}{5.12} & \multicolumn{2}{|c|}{1.28} \\
\hline \multirow[t]{2}{*}{$n=3, L=2$} & \multicolumn{6}{|c|}{$\mathrm{CT}-\mathrm{I} \Sigma \Delta_{(3,2)}, \mathrm{M}=32$} \\
\hline & $\mathrm{INT}_{11}$ & $\mathrm{INT}_{12}$ & $\mathrm{INT}_{21}$ & $\mathrm{INT}_{22}$ & $\mathrm{INT}_{31}$ & $\mathrm{INT}_{32}$ \\
\hline GBW (k Hz) & 204.8 & 102.4 & 92.16 & 40.96 & 40.96 & 20.49 \\
\hline DC Gain [dB] & 40 & 30 & 30 & 20 & 20 & 10 \\
\hline $\mathrm{SR}\left[10^{5} \mathrm{~V} / \mathrm{s}\right]$ & 2.05 & 1.02 & 0.92 & 0.41 & 0.41 & 0.20 \\
\hline
\end{tabular}

more power and area consumption. In addition, the required conversion resolution and speed should be taken into account in determining an optimal solution.

\section{Design StRategy}

In this section, we aim to search for a power-conscious, area-efficient solution, assuming a 14-bit target resolution. According to Fig. 2, the two configurations with $n=1$, which are essentially single-stage $\mathrm{I} \Sigma \Delta$ ADCs, require large $\mathrm{M}$ and thus high sampling rate to achieve high-resolution. So, only the pipeline configurations (with $n=2$ and $n=3$ ) are selected for further comparison. Non-idealities in the integrator's amplifier, i.e., finite amplifier gain-bandwidth-product (GBW), DC gain and slew-rate (SR), are modeled assuming an active-RC implementation. Table II shows the derived amplifier related specifications when targeting a signal-to-noise-plus-distortionratio (SNDR) of $86-\mathrm{dB}$ over $3.2-\mathrm{kHz}$ signal bandwidth.

To quantify the total power consumption of each configuration, power efficient circuit blocks are exploited to implement the active blocks. In all the configurations, the first integrator $\mathrm{INT}_{11}$, dominates the noise and linearity performance of the entire ADC. In addition, the large integrating capacitor in INT $_{11}$ (due to a small scaling coefficient $c_{1}$ ) loads the amplifier significantly and requires a large current drive capability. A Class-A/Class-AB configuration with two independent common-mode feedback (CMFB) loops is used to implement the first amplifier [6]. According to Table II, specifications are found much relaxed for the amplifiers in a later active block. So, simple inverter-based amplifiers can be employed to implement the other active blocks. Inverter-based amplifiers in DT $\Sigma \Delta$ modulators have been widely investigated to improve the power- and area- efficiency [7], [8]. One drawback of a simple inverter is that its quiescent current is undefined and strongly influenced by process-voltage-temperature (PVT) 


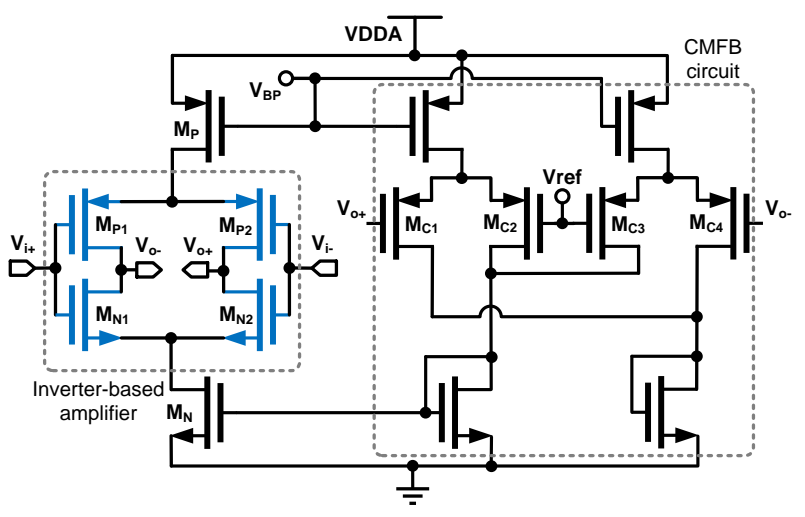

Fig. 3: Inverter-based amplifier.

TABLE III: Estimated power consumption.

\begin{tabular}{lll}
\hline \hline $\mathrm{P}_{(\mathrm{n}, \mathrm{L})}$ & Power consumption contributors & Power \\
\hline $\mathrm{P}_{(2,2)}$ & $\mathrm{P}_{\mathrm{INT}_{11}}+\mathrm{P}_{\mathrm{INT}_{12}}+\mathrm{P}_{\mathrm{INT}_{21}}+\mathrm{P}_{\mathrm{INT}_{22}}+2 \mathrm{P}_{\mathrm{C}}$ & $19.08 \mu \mathrm{W}$ \\
\hline $\mathrm{P}_{(2,3)}$ & $\mathrm{P}_{\mathrm{INT}_{11}}+\mathrm{P}_{\mathrm{INT}_{12}}+\mathrm{P}_{\mathrm{INT}_{13}}+\mathrm{P}_{\mathrm{INT}_{21}}+\mathrm{P}_{\mathrm{INT}_{22}}+\mathrm{P}_{\mathrm{INT}_{23}}+2 \mathrm{P}_{\mathrm{C}}$ & $19.75 \mu \mathrm{W}$ \\
\hline $\mathrm{P}_{(3,1)}$ & $\mathrm{P}_{\mathrm{INT}_{11}}+\mathrm{P}_{\mathrm{INT}_{12}}+\mathrm{P}_{\mathrm{INT}_{13}}+3 \mathrm{P}_{\mathrm{C}}$ & $40.54 \mu \mathrm{W}$ \\
\hline $\mathrm{P}_{(3,2)}$ & $\mathrm{P}_{\mathrm{INT}_{11}}+\mathrm{P}_{\mathrm{INT}_{12}}+\mathrm{P}_{\mathrm{INT}_{21}}+\mathrm{P}_{\mathrm{INT}_{22}}+\mathrm{P}_{\mathrm{INT}_{31}}+\mathrm{P}_{\mathrm{INT}_{32}}+3 \mathrm{P}_{\mathrm{C}}$ & $19.88 \mu \mathrm{W}$ \\
\hline \hline \multicolumn{2}{c}{ Note: } & $\mathrm{P}_{\mathrm{C}}$ is the averaged dynamic power of the multi-input comparator.
\end{tabular}

variations [8]. To compensate for this issue, design techniques, such as auto-zeroing [7] and LDO biasing [8], have been developed for DT inverter-based $\Sigma \Delta$ modulators. In this work, a robust design for $\mathrm{CT}$ implementation is achieved by using a current mirror to control precisely the DC operating point of a fully-differential inverter as shown in Fig. 3. In this way, the transconductance of $\mathrm{M}_{\mathrm{P} 1} / \mathrm{M}_{\mathrm{N} 1}$ and $\mathrm{M}_{\mathrm{P} 2} / \mathrm{M}_{\mathrm{N} 2}$ can be set by adjusting the biasing current through $M_{P} . M_{P}$ and $M_{N}$ are also used to compensate for the uncertainties in the gate-tosource voltages of $\mathrm{M}_{\mathrm{P} 1} / \mathrm{M}_{\mathrm{N} 1}$ and $\mathrm{M}_{\mathrm{P} 2} / \mathrm{M}_{\mathrm{N} 2}$. In addition, a CMFB circuit is utilized to regulate the output common-mode level to be less dependent on process variation.

For CT loop filter with a CIFF-IFF topology, a dedicated summing amplifier is usually adopted to perform the weighted summation of feed-forward paths. To save power and area, the weighted summation is integrated into a multi-input dynamic comparator [9]. As shown in Fig. 4, the two integrators' outputs $\left(\mathrm{X}_{1 \pm}, \mathrm{X}_{2 \pm}\right)$ as well as the input signal $\left(\mathrm{IN}_{ \pm}\right)$are connected to the differential inputs of the comparator. In this way, the feed-forward summation can be applied with virtually no additional power and area. Moreover, to minimize the kickback noise of this dynamic comparator, a neutralization technique [10] is applied. As shown in Fig. 4, two crosscoupled capacitances, which are formed by source-and-drainconnected $\mathrm{M}_{\mathrm{a}}$ and $\mathrm{M}_{\mathrm{b}}$, are added to the input pair $\mathrm{M}_{1}$ and $\mathrm{M}_{2}$, to compensate for their gate-to-drain capacitances.

Table III provides the estimated power consumption when the proposed circuit blocks are used. It can be seen that there is a very slight difference in the total power consumption among the CT-I $\Sigma \Delta_{(2,2)}$, CT-I $\Sigma \Delta_{(2,3)}$ and CT-I $\Sigma \Delta_{(3,2)}$ configurations. On the other hand, the CT-I $\Sigma \Delta_{(2,2)}$ occupies the minimum area

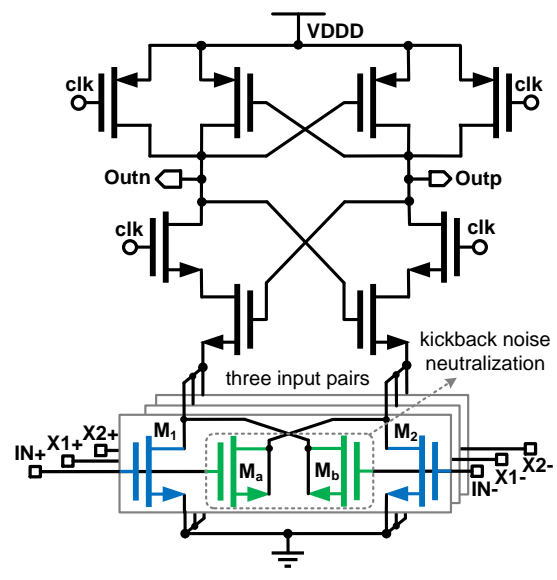

Fig. 4: Multi-input dynamic comparator.

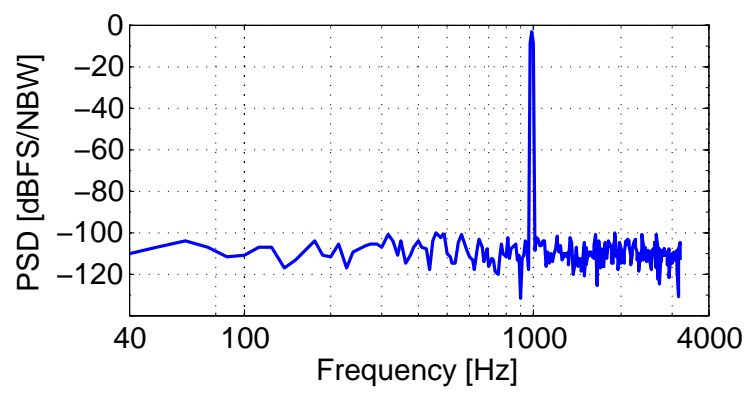

Fig. 5: PSD of the test-case ADC for a $-3.1 \mathrm{~dB}_{\mathrm{FS}}$ input signal at $987.5-\mathrm{Hz}$. A 512-point FFT with Hann window is applied.

as it requires four integrators while the other two require six. Therefore, as for the target resolution and signal bandwidth, the CT-I $\Sigma \Delta_{(2,2)}$ is the optimal solution. When it comes to a higher resolution or a wider signal bandwidth, the CT-I $\Sigma \Delta_{(2,3)}$ and CT-I $\Sigma \Delta_{(3,2)}$ might be better alternatives.

\section{CASE Study}

Based on the previous discussion and considering the target specifications, the CT-I $\Sigma \Delta_{(2,2)}$ configuration is chosen as an implementation case study. To leave design margins accounting for noise and other non-idealities, $M=50$ is selected, which corresponds to a $320-\mathrm{kHz}$ clock frequency in the $\Sigma \Delta$ modulators. The circuits are implemented in a $0.18-\mu \mathrm{m}$ CMOS technology, and consume a total current of $15.6-\mu \mathrm{A}$ from a $1.2-\mathrm{V}$ power supply. Specifically, $13.51-\mu \mathrm{A}$ is consumed in the $\mathrm{INT}_{11}, 875-\mathrm{nA}$ in the $\mathrm{INT}_{12}, 729-\mathrm{nA}$ in the $\mathrm{INT}_{21}, 327-$ $\mathrm{nA}$ in the $\mathrm{INT}_{22}$, and $107-\mathrm{nA}$ in each comparator. Post-layout transient-noise simulation shows that a peak SNDR of 82.5$\mathrm{dB}$ is achieved for a $-3.1 \mathrm{~dB}_{\mathrm{FS}}$ sinusoidal input at 987.5Hz. Fig. 5 shows the corresponding power spectrum density (PSD) plot. In order to evaluate the ADC's sensitivity to PVT variations, extensive circuit-level simulations have been performed. Monte-Carlo simulations have been performed under the influence of both process variation and device mismatch. A histogram showing the distribution of the SNDR is presented in Fig. 6, demonstrating a mean SNDR of $86.45 \mathrm{~dB}$. It also 


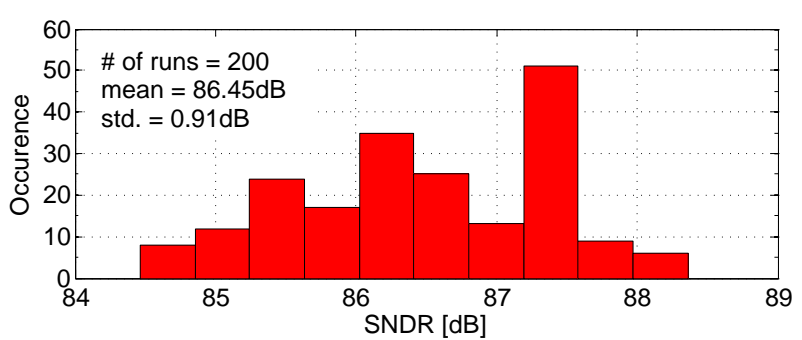

Fig. 6: Histogram of the SNDR performance obtained from 200 runs of Monte-Carlo simulations under the influence of process variation and device mismatch.
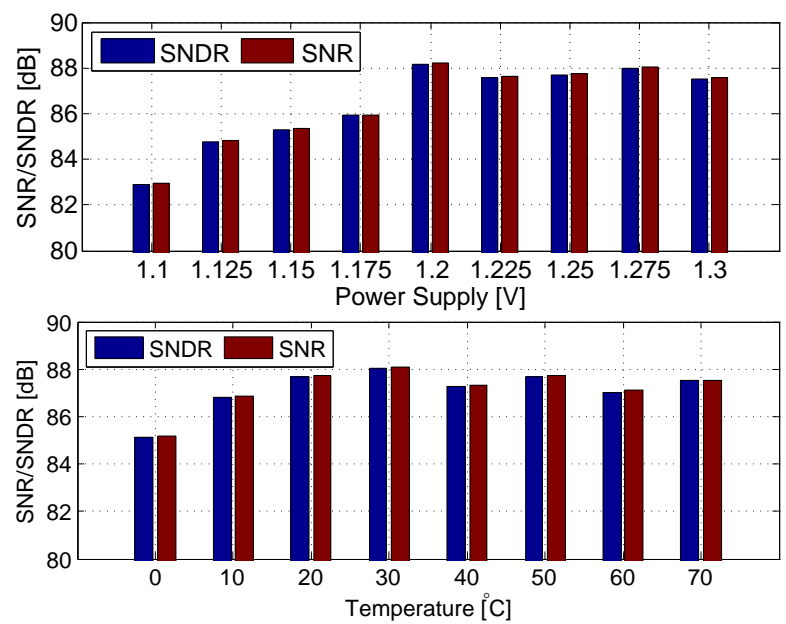

Fig. 7: Simulated SNR/SNDR over power supply and temperature variations.

TABLE IV: Post-layout Simulation Performance Summary

\begin{tabular}{ll}
\hline \hline Signal Bandwidth & $3.2 \mathrm{kHz}$ \\
\hline Sampling Frequency & $320 \mathrm{kHz}$ \\
\hline SFDR & $96.9 \mathrm{~dB}$ \\
\hline peak SNDR & $82.5 \mathrm{~dB}$ \\
\hline Power Consumption & $18.27 \mu \mathrm{W}$ \\
\hline Supply Voltage & $1.2 \mathrm{~V}$ \\
\hline Technology & $0.18 \mu \mathrm{m}$ \\
\hline Active Area & $0.207 \mathrm{~mm}^{2}$ \\
\hline FOM & $268.6 \mathrm{fJ} / \mathrm{conv}$ \\
\hline \hline
\end{tabular}

shows that in approximately $95 \%$ of the cases, the SNDR is larger than 85-dB. Fig. 7 shows the simulated SNR/SNDR over $\pm 10 \%$ variations on the power supply and a commercial temperature range of $0^{\circ} \mathrm{C}-70^{\circ} \mathrm{C}$.

A summary of the simulated performance is presented in Table IV. The figure-of-merit (FOM) of this design is compared to the state-of-the-art I $\Sigma \Delta$ prototype ADCs, as shown in Fig. 8. The FOM achieved by this work, which is taken from post-layout simulation, indicates that the test-case $\mathrm{ADC}$ is a very promising solution for the power- and areaconstrained biosensor applications.

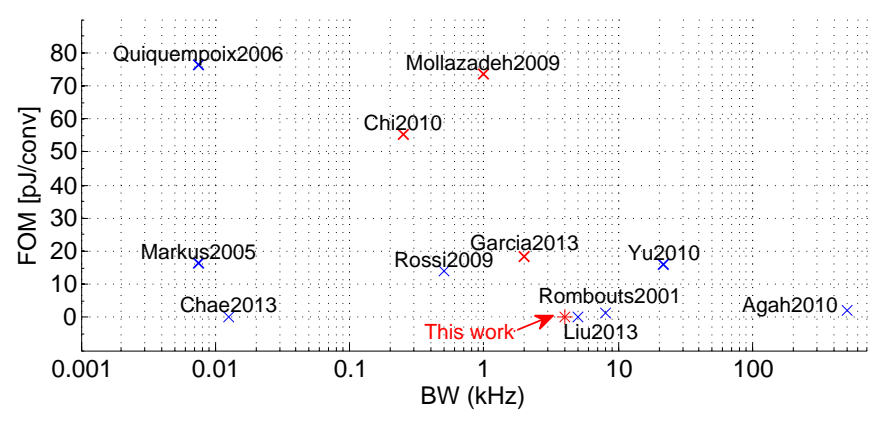

Fig. 8: $\mathrm{FOM}^{+}$comparison with existing I $\Sigma \Delta$ prototype ADCs. Experimental results: CT I $\Sigma \Delta$ ADCs $(\times)$ and DT I $\Sigma \Delta$ ADCs $(\times)$; Post-layout simulation results: CT-I $\Sigma \Delta_{(2,2)}(*)$.

${ }^{+}$The FOM is calculated as: $\mathrm{FOM}=\mathrm{P} /\left(2 \times \mathrm{BW} \times 2^{\mathrm{ENOB}}\right)$.

\section{CONCLUSION}

A power-efficient pipelined ADC architecture, which employs CT I $\Sigma \Delta$ ADC stages, has been proposed in this paper. A power-conscious, area-efficient design strategy has been used in searching for an optimal combination of design parameters that results in minimized power-area consumption. Inverterbased amplifiers have been explored and power-efficient comparators have been employed leading to further reduction in power and area. An implementation test-case, which follows the design strategy, has been presented. The post-layout simulation shows an FOM of 268.6 fJ/conv.

\section{ACKNOWLEDGMENT}

This work has been supported by Swedish Research Council (VR) under the erBio project.

\section{REFERENCES}

[1] J. Markus, J. Silva, and G. C. Temes, "Theory and applications of incremental $\Delta \Sigma$ converters," IEEE Trans. Circuits Syst. I, vol. 51, no. 4 pp. 678-690, 2004.

[2] V. Quiquempoix, P. Deval, A. Barreto, G. Bellini, J. Markus, J. Silva, and G. C. Temes, "A low-power 22-bit incremental ADC," IEEE J. Solid-State Circuits, vol. 41, no. 7, pp. 1562-1571, 2006.

[3] A. Agah, K. Vleugels, P. B. Griffin, M. Ronaghi, J. D. Plummer, and B. A. Wooley, "A high-resolution low-power incremental $\Sigma \Delta$ ADC with extended range for biosensor arrays," IEEE J. Solid-State Circuits, vol. 45, no. 6, pp. 1099-1110, 2010.

[4] J. Garcia, S. Rodriguez, and A. Rusu, “A Low-Power CT Incremental 3rd Order Sigma-Delta ADC for Biosensor Applications," IEEE Trans. Circuits Syst. I, vol. 60, no. 1, pp. 25-36, 2013.

[5] S. Tao, S. Rodriguez and A. Rusu, "Two-step continuous-time incremental sigma-delta ADC," in IET Electron. Lett., vol. 49, no. 12, pp. 749-751, 2013.

[6] S. Pavan, N. Krishnapura, R. Pandarinathan, and P. Sankar, "A Power Optimized Continuous-Time $\triangle \Sigma$ ADC for Audio Applications," IEEE J. Solid-State Circuits, vol. 43, no. 2, pp. 351-360, 2008.

[7] Y. Chae and G. Han, "Low Voltage, Low Power, Inverter-Based Switchedcapacitor Delta Sigma Modulator," IEEE J. Solid-State Circuits, vol. 44 no. 2, pp. 458-472, 2009

[8] T. Christen, "A 15-bit 140- $\mu \mathrm{W}$ Scalable-Bandwidth Inverter-Based $\Delta \Sigma$ Modulator for a MEMS Microphone with Digital Output," IEEE J. SolidState Circuits, vol. 48, no. 7, pp. 1605-1614, 2013.

[9] J. Zhang, Y. Lian, L. Yao, and B. Shi, "A 0.6-V 82-dB 28.6- $\mu \mathrm{W}$ Continuous-Time Audio Delta-Sigma Modulator," IEEE J. Solid-State Circuits, vol. 46, pp. 2326-2335, 2011.

[10] P. M. Figueiredo and J. C. Vital, "Kickback noise reduction techniques for CMOS latched comparators, " IEEE Trans. Circuits Syst. II, vol. 53, no. 7, pp. 541-545, 2006. 